Editorial

\title{
Proteomics advancements in dentistry
}

\section{Abbreviations}

PCR, polyacrylamide; MS, mass spectrometry; AEP, acquired enamel pellicle; SKB, salivaomics knowledge base; PDL, periodontal ligaments

\section{Editorial}

Proteins are enormously composed of sophisticated 20 different types of amino acids. Proteins perform a crucial role in structural support, catalysis, and signal transmission, hence called "working horses" of a cell. ${ }^{1}$ The word "Proteome" is amalgamation of two words "protein" and "genome", were first introduced by Mark Wilkins in 1986. ${ }^{2}$ Proteomics is the special branch of life sciences that deals with the distribution and interaction of proteins in time, and space in cells, organisms or even within an ecosystem. For this contents of proteins sample measure through a sequence of separation, identification and in last counted by highly specialized proteomics tools such as polyacrylamide gel electrophoresis (2D-Gel), mass spectrometry (MS), real time-PCR, microarrays and chromatographical tools. ${ }^{3-5}$ This discovery science brings revolution in biomedical sciences by revealing the normal physiological and pathological phenomenon occurring in living organisms.

In last two decades, proteomics technologies widely used in dentistry and produced huge amount of protein data regarding physiological conditions and pathological conditions. ${ }^{6-10}$ In medical science literature electronic databases (e.g. PubMed, Scopus, and Google Scholar) search reported $60 \%$ to $70 \%$ use of different proteomical tools in normal or diseased dental tissues (enamel, dentin, pulp, cementum, alveolar bone, saliva, gingival crevicular fluids, acquired enamel pellicle, and oral mucosa) and dental materials for proteomic analysis. ${ }^{11-16}$ Siqueira et al. ${ }^{17}$ identified human acquired enamel pellicle (AEP) proteins composition through LC-ESIMS/MS and detected 130 total proteins. ${ }^{17}$ AEP provide selective permeability barrier that controls demineralization/remineralization and act as lubricating film, and its proteome configuration can help in understanding of dental decay processes..$^{18}$ Human whole mouth saliva is analysed frequently through proteomic tools due to its noninvasive, patient privacy control, and easy sampling. Approximately 3000 salivary proteome are reported in Salivaomics Knowledge Base (SKB), developed by the UCLA School of Dentistry, USA (www.hspp. ucla.edu/skb.swf). ${ }^{19}$ Dame et al. ${ }^{20}$ used a variety of analytical tools including gas chromatography mass spectrometry, nuclear magnetic resonance spectroscopy, direct flow injection/liquid chromatography mass spectrometry, inductively coupled plasma mass spectrometry and high performance liquid chromatography to calculate 308 salivary metabolites..$^{20}$ More recently, Mrozik et al. ${ }^{21}$ evaluated the comparison of proteome between bone marrow, periodontal ligaments (PDL) and dental pulp through 2-DE, MS/MS. ${ }^{21}$ Salmon et al. ${ }^{22}$ comprehensively analysed proteomics of dental cementum through LC-MS/MS and detected 231 proteins, very interestingly researcher identified superoxide dismutase-3, a novel biomarker associated with cementum and cementum cells. ${ }^{22}$

To sum up, proteomics tools bring drastic change in diagnosis, treatment and prevention of dental diseases. With the help of
Volume 4 Issue 2 - 2016

\author{
Zohaib Khurshid,' Sana Zohaib, ${ }^{2}$ Shariq \\ Najeeb, ${ }^{3}$ Muhammad Sohail Zafar ${ }^{4}$ \\ 'Department of Biomaterials, UK \\ ${ }^{2}$ Department of Biomedical Engineering, Saudi Arabia \\ ${ }^{3}$ Restorative Dental Sciences, Saudi Arabia \\ ${ }^{4}$ Department of Restorative Dentistry, Saudi Arabia
}

Correspondence: Muhammad Sohail Zafar, Department of Restorative Dentistry, College of Dentistry, Taibah University, Saudi Arabia,

Emaildrsohail_78@hotmail.com,mzafar@taibau.edu.sa

Received: January 27, 20I5 | Published: February 16, 2016

proteomic tools dentistry getting specialized field which deals disease condition with minimal interventions.

\section{Funding}

None.

\section{Acknowledgements}

None.

\section{Conflicts of interest}

Authors declare that there is no conflict of interest.

\section{References}

1. Alberts B. The Cell as a Collection of Protein Machines: Preparing the Next Generation of Molecular Biologists. Cell. 1998;92(3):291-294.

2. Bhattacharyya M. Protein Structure and Function: Looking through the Network of Side-Chain Interactions. Curr Protein Pept Sci. 2015;17(1):4 25.

3. Gama MR, Collins CH, Bottoli CBG. Nano-liquid chromatography in pharmaceutical and biomedical research. $J$ Chromatogr Sci. 2013;51(7):694-703.

4. Gstaiger M, Aebersold R. Applying mass spectrometry-based proteomics to genetics, genomics and network biology. Nat Rev Genet. 2009;10(9):617-627.

5. Kinoshita E, Kinoshita-Kikuta E, Koike T. The Cutting Edge of Affinity Electrophoresis Technology. Proteomes. 20153(1):42-55.

6. Garg K, Chandra S, Raj V, et al. Molecular and genetic aspects of odontogenic tumors: a review. Iran J Basic Med Sci. 2015;18(6):529-536.

7. Khurshid Z, Naseem M, Sheikh Z, et al. Oral antimicrobial peptides: Types and role in the oral cavity. Saudi Pharmaceutical Journal. 2015;24(5):515-524.

8. Khurshid Z, Zafar M, Qasim S, et al. Advances in Nanotechnology for Restorative Dentistry. Materials. 2015;8(2):717-731. 
9. Zafar MS, Khurshid Z, Almas K. Oral tissue engineering progress and challenges. Tissue Engineering and Regenerative Medicine. 2015;12(6):387-397.

10. Sheikh Z, Abdallah M, Hamdan N. Barrier Membranes for Periodonta Guided Tissue Regeneration Applications. 2014;6.

11. Hubbard MJ, Kon JC. Proteomic analysis of dental tissues. J Chromatogr. 2002;B 771(1-2):211-220.

12. Huynh AHS, Veith PD, McGregor NR, et al. Gingival crevicular fluid proteomes in health, gingivitis and chronic periodontitis. J Periodontal Res. 2014.

13. Jágr M, Eckhardt A, Pataridis S, et al. Proteomics of Human Teeth and Saliva. Physiol Res. 2014;63(Suppl 1):S141-S154.

14. Slavkin HC, Fox CH, Meyer DM. Salivary Diagnostics and Its Impac in Dentistry, Research, Education, and the Professional Community. Adv Dent Res. 2011;23(4):381-386.

15. Farges JC, Alliot Licht B, Renard E, et al. Dental Pulp Defence and Repair Mechanisms in Dental Caries. Mediators Inflamm. 2015;230251.
16. Fuentes L, Yakob M, Wong DTW Emerging horizons of salivary diagnostics for periodontal disease. Br Dent J . 2014;217(10):567-573.

17. Siqueira W, Zhang W, Helmerhorst EJ, Gygi S, Oppenheim FG. Identification of protein components in in-vivo human acquired enamel pellicle using LC-ESI-MS/MS. J Proteome Res. 2007;6(6):2152-2160.

18. Siqueira WL, Helmerhorst EJ, Zhang W, et al. Acquired enamel pellicle and its potential role in oral diagnostics. Ann N Y Acad of Sciences. 2007;1098:504-509.

19. Wong DT. Salivary diagnostics powered by nanotechnologies, proteomics and genomics. J Am Dent Assoc. 2006;137(3):313-321.

20. Dame ZT, Aziat F, Mandal R, et al.The human saliva metabolome. Metabolomics. 2015;11(6):1864-1883.

21. Mrozik KM, Zilm PS, Bagley CJ, et al. Proteomic characterization of mesenchymal stem cell-like populations derived from ovine periodontal ligament, dental pulp, and bone marrow: analysis of differentially expressed proteins. Stem Cells Dev. 2010;19(10):1485-1499.

22. Salmon CR, Tomazela DM, Ruiz KGS, et al. Proteomic analysis of human dental cementum and alveolar bone. J Proteomics. 2013;91:544-555. 